\title{
A Characterization of Stochastic Independence by Association, with an Application to Random Utility Theory
}

\author{
Hans Colonius \\ Technische Universität Braunschweig
}

\begin{abstract}
A characterization of stochastic independence in terms of association of random variables is given. The result is applied to yield a simple proof of the Sattath-Tversky inequality without continuity assumptions.
\end{abstract}

Several concepts of positive dependence of random variables have been studied by Lehmann (1966) in the bivariate case. Esary, Proschan, and Walkup (1967) introduced the multivariate concept of "association" and demonstrated a number of useful applications. Reviews are given in Tong (1980) and Eaton (1982). In this paper, a new characterization of independence is given in terms of association. Subsequently, the result is used to yield a simple proof, in a more general setting, of the Sattath-Tversky inequality in random utility (Sattath \& Tversky, 1976).

Following Esary et al. (1967), an $n$-variate random variable $X$ is, or equivalently, the coordinate random variables $X_{1}, \ldots, X_{n}$ are associated if

$$
\operatorname{cov}|f(X), g(X)| \geqslant 0
$$

for all nondecreasing realvalued functions $f$ and $g$ for which the above covariance exists (here, a realvalued function on a subset of $R^{n}$ is called nondecreasing (nonincreasing) if it is so in each of its arguments separately). Association captures the intuition of "positive dependence" of random variables. For example, it can be shown that for associated random variables $X_{1}, \ldots, X_{n}$

$$
P\left(X_{i} \geqslant x_{i}, i=1, \ldots, n\right) \geqslant \prod_{i=1}^{n} P\left(X_{i} \geqslant x_{i}\right)
$$

holds for all $x_{1}, \ldots, x_{n}$ (see, e.g., Esary et al., 1967). Proofs of the following useful properties of association can be found in the references cited above (for (P6) see Ncwman \& Wright, 1981).

Requests for reprints should be sent to the author at Technische Universität Braunschweig, Institut für Psychologie, Spielmannstrasse 19, D-3300 Braunschweig, Federal Republic of Germany. 
(P1) Any subset of associated random variables is a set of associated random variables.

(P2) The set consisting of a single random variable is associated.

(P3) If two sets of associated random variables are independent, then their union is a set of associated random variables.

(P4) If $\operatorname{cov}[u(X), v(X)]$ is nonnegative for all pairs of binary nondecreasing functions $u$ and $v$, then $X$ is also associated (by definition, a binary function takes only the value 0 or 1 ).

(P5) Nondecreasing functions of associated random variables are associated random variables.

(P6) Associated random variables that are pairwise uncorrelated are jointly independent.

It easily follows from (P2) and (P3) that independent random variables are associated. The following definition will be central to a characterization of independence by association.

DEFINITION. An $n$-variate random variable $X$, or equivalently, the coordinate random variables $X_{1}, \ldots, X_{n}$ are said to satisfy the $A I S$ condition (association independent of sign) if the following holds:

(AIS) $(-1)^{j_{1}} X_{1}, \ldots,(-1)^{j_{n}} X_{n}$ are associated for $j_{i}=0$ or $1, \quad i=1, \ldots, n$.

The lemma below indicates a condition equivalent to AIS. (In that lemma, two realvalued functions on a subset of $R^{n}$ are called concordant if they are monotone, i.e., nondecreasing or nonincreasing, in each argument and if the direction of montonicity in each argument is the same for both functions; cf. Lehmann, 1966.)

LEMMA 1. An n-variate random variable satisfies the AIS condition if and only if $E q$. (1) holds for all pairs of concordant functions $f$ and $g$ for which the covariance exists.

Since any function $f$ that is nonincreasing in $x_{i}$ is nondecreasing in $-x_{i}$ and vice versa, the proof of the above lemma is obvious.

LEMMA 2. (P1)-(P4) hold true with "associated" replaced by "satisfying the AIS condition" and with "nondecreasing" replaced by "concordant" in (P4).

The proof of this lemma is immediate from an inspection of the corresponding proofs in Esary et al. (1967) and will be omitted here. In particular, the lemma implies that independent random variables satisfy AIS. Incidentally, the latter fact also follows from a theorem in Jogdeo (1977) where the AIS condition was not stated explicitly. The following theorem establishes the equivalencc announced earlier.

THEOREM. Let $X_{1}, \ldots, X_{n}(n \geqslant 2)$ be finite variance random variables on some 
probability space; then $X_{1}, \ldots, X_{n}$ are independent if and only if they satisfy the AIS condition.

Proof. The implication independence $\rightarrow$ AIS follows from Lemma 2. For the other direction, suppose $X_{1}, \ldots, X_{n}$ satisfy AIS; by (P1), $\left\{X_{i}, X_{j}\right\}$ and $\left\{X_{i},-X_{j}\right\}$ are associated, respectively, for any $i, j \in\{1, \ldots, n\}$. From the definition of association, we have specifically

$$
\operatorname{cov}\left(X_{i}, X_{j}\right) \geqslant 0 \quad \text { and } \quad \operatorname{cov}\left(X_{i},-X_{j}\right) \geqslant 0
$$

thus $\operatorname{cov}\left(X_{i}, X_{j}\right)=0$; then (P6) implies independence of $X_{1}, \ldots, X_{n}$ which ends the proof.

Remarks. (1) Another way to prove the implication AIS $\rightarrow$ independence without referring to (P6) is possible using Lemma 1.

(2) A related result can be found in Jogdeo (1968) where independence is characterized by Eq. (2), uncorrelatedness, and the condition $E\left(X_{1} \cdots X_{n}\right)=$ $E\left(X_{1}\right) \cdots E\left(X_{n}\right)$.

One way to utilize the above characterization of independence is to generalize a probability inequality pertinent to random utility theory (cf. Luce \& Suppes, 1965). Let $X_{1}, \ldots, X_{n}$ be independent random variables; for any $i \in I \cap J \subset\{1, \ldots, n\}$, the inequality

$$
\begin{aligned}
P\left(X_{i} \geqslant\right. & \left.\max \left(X_{j} \mid j \in I \cup J\right)\right) \geqslant P\left(X_{i} \geqslant \max \left(X_{j} \mid j \in I\right)\right) \\
& \times P\left(X_{i} \geqslant \max \left(X_{j} \mid j \in J\right)\right)
\end{aligned}
$$

was shown to hold by Sattath and Tversky (1976) if the random variables are continuous. In view of Lemma 1, the general case, i.e., without continuity assumption, follows immediately from defining concordant functions $f$ and $g$ by

$$
\begin{array}{rlrl}
f\left(X_{1}, \ldots, X_{n}\right)=1 & \text { if } & X_{i} \geqslant \max \left(X_{j} \mid j \in I\right), \\
=0 & \text { if } \quad \text { else, } \\
g\left(X_{1}, \ldots, X_{n}\right)=1 & \text { if } \quad X_{i} \geqslant \max \left(X_{j} \mid j \in J\right), \\
=0 & \text { if } \quad \text { else. }
\end{array}
$$

A paper investigating the concept of association in random utility theory is in preparation.

\section{REHERENCES}

Esary, J. D., Proschan, F., \& Walkup, D. W. Association of random variables with applications. The Annals of Mathematical Statistics, 1967, 38, 1466-1474. 
EAton, M. L. A review of selected topics in multivariate probability inequalities. The Annals of Statistics, 1982, 10, 11-43.

JoGDEO, K. Characterizations of independence in certain families of bivariate and multivariate distributions. The Annals of Mathematical Statistics, 1968, 39, 433-441.

JoGdeo, K. Association and probability inequalities. The Annals of Statistics, 1977, 5, 495-504.

Lehmann, E. L. Some concepts of dependence. The Annals of Mathematical Statistics, 1966, 37. $1137-1152$.

LucE, R. D., \& Suppes, P. Preference, utility and subjective probability. In R. D. Luce, R. R. Bush, \& E. Galanter (Eds.), Handbook of mathematical psychology, Vol. III. New York: Wiley, 1965.

Newman, C. M., \& Wright, A. L. An invariance principle for certain dependent sequences. The Annals of Probability, 1981, 9, 671-675.

SATtATH, S., \& TVERSKY, A. Unite and conquer : A multiplicative inequality for choice probability. Econometrica, 1976, 44, 79-89.

Tong, Y. L. Probability inequalities in multivariate distributions. New York: Academic Press, 1980.

RECEIVED: February 18, 1983 